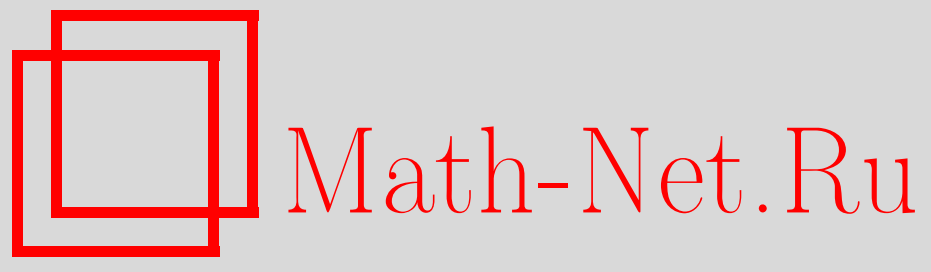

В. П. Маслов, Теорема о парастатистиках и ее приложение, ТМФ, 2007, том 150, номер 3, 511-512

DOI: https://doi.org/10.4213/tmf5993

Использование Общероссийского математического портала Math-Net.Ru подразумевает, что вы прочитали и согласны с пользовательским соглашением http://www . mathnet.ru/rus/agreement

Параметры загрузки:

IP: 3.89 .197 .203

26 апреля 2023 г., $12: 56: 48$

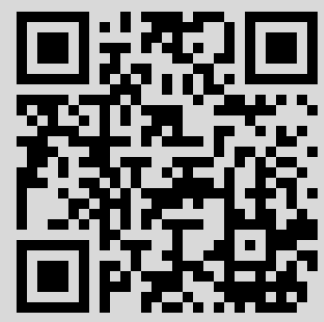




\section{ТЕОРЕМА О ПАРАСТАТИСТИКАХ И ЕЕ ПРИЛОЖЕНИЕ}

Из условия, что природа выбирает безрисковую стратегию, как следствие получено распределение по парастатистикам.

Ключевые слова: парастатистики, безрисковая стратегия, концепция "отсутствия интеллекта".

Прежде всего заметим, что природа выбирает безрисковую ситуацию. Если известно, что общее число тождественных частиц не превосходит некоторой величины и расставлены приоритеты поглощающих эти частицы веществ (например, согласно таблице Менделеева), то приводимая ниже теорема может быть применима к такого рода физическим моделям. Поскольку денежные купюры одного достоинства обладают свойством тождественности (см. [1]), то мы будем говорить на языке финансовой математики, поскольку в финансовой математике безрисковое вложение капитала является задачей особенно актуальной. Приложение теоремы к физическим объектам мы оставим читателю.

Предположим, что предприятия, в которые инвестор хочет сделать вложения, выбираются самим инвестором. Инвестор также устанавливает порядок приоритетов среди этих предприятий и указывает бюджетное ограничение и ограничение вида (1) (см. ниже). Тогда нужно разложить деньги по этим предприятиям так, чтобы операционный риск был минимальным.

В решении этой задачи мы будем придерживаться концепции “отсутствия интеллекта" (zero intelligence) для финансовых рынков [2]-[4]. Поэтому если мы найдем наибольшее "скопление" вариантов для нашей задачи, которые равновероятны в силу принципа "отсутствия интеллекта", то именно такое разложение будет наименее опасным, безрисковым.

Пусть согласно условию величина $C_{i}$ (безразлично в какой валюте) вложения в $i$-е предприятие удовлетворяет соотношению

$$
C_{i}+R \geqslant C_{i+1} \geqslant C_{i}, \quad i=1,2, \ldots, s-1 ; \quad C_{s}=M, \quad C_{1}=K,
$$

причем $M$ и $K$ заданы. Отсюда $C_{s}-C_{1}=M-K$. Поэтому бюджетное ограничение $\Phi$ удовлетворяет соотношению

$$
s K \leqslant \sum_{i=1}^{s} C_{i} \leqslant \Phi \leqslant M s .
$$

\footnotetext{
*Московский государственный университет, Москва, Россия. E-mail: v.p.maslov@mail.ru
} 
Имеем

откуда получаем

$$
\sum_{i=1}^{s} C_{i} \equiv \sum_{i=2}^{s}(s-i)\left(C_{i}-C_{i-1}\right)+C_{1} s \leqslant \Phi
$$

$$
\sum_{i=2}^{s}(s-i)\left(C_{i}-C_{i-1}\right) \leqslant \Phi-K s .
$$

Обозначив $n_{i}=C_{i}-C_{i-1}, \lambda_{i}=s-i, \Phi^{\prime}=\Phi-K s$, имеем

$$
\sum_{j=2}^{i} n_{j}=C_{i}-C_{1}
$$

Мы получаем

$$
\sum_{i=2}^{s} n_{i} \lambda_{i} \leqslant \Phi^{\prime}, \quad \sum_{j=2}^{s} n_{j}=M-K .
$$

Поскольку все наборы $\left\{C_{i}\right\}$ равновероятны при условиях (1) и (2), то отсюда следует, что значения $n_{i}$, удовлетворяющие равенству $\sum_{i=2}^{s} n_{i}=M-K$, равновероятны при условии $R \geqslant n_{i} \geqslant 0$ и условии (5).

ТЕОРЕМА О ПАРАСТАТИСТИКАХ. Число вариантов $\left\{n_{i}\right\}, i=2, \ldots, s$, таких, что выполнены условия (5), и для которых

$$
\left|C_{i}-C_{1}-\sum_{j=2}^{i}\left\{\frac{1}{e^{\beta(s-j)-\sigma}-1}-\frac{R+1}{e^{\beta(R+1)(s-j)-(R+1) \sigma}-1}\right\}\right| \geqslant s^{\frac{1}{4}+\varepsilon},
$$

менъше, чем $(s / L)^{-d}$, где $d$ - любое число, $L$ - общее число вариантов, $i>\varepsilon s, \varepsilon$ сколь угодно мало.

Константы $\sigma$ и $\beta$ находятся из условий

$$
\begin{gathered}
M-K=\sum_{j=2}^{s}\left\{\frac{1}{e^{\beta(s-j)-\sigma}-1}-\frac{R+1}{e^{\beta(R+1)(s-j)-(R+1) \sigma}-1}\right\}, \\
\sum_{j=2}^{s}\left\{\frac{s-j}{e^{\beta(s-j)-\sigma}-1}-\frac{(s-j)(R+1)}{e^{\beta(R+1)(s-j)-(R+1) \sigma}-1}\right\}=\Phi^{\prime}=\Phi-K s .
\end{gathered}
$$

Аналогичная теорема доказывается в работах [5]-[7].

СлЕДСТВИЕ. Безрисковое вложение при условиях (1), (2) осуществляется, если

$$
C_{i}=C_{1}+\sum_{j=2}^{i}\left\{\frac{1}{e^{\beta(s-j)-\sigma}-1}-\frac{R+1}{e^{\beta(R+1)(s-j)-(R+1) \sigma}-1}\right\} .
$$

\section{Список литературы}

[1] В. П. Маслов, Квантовая экономика, Наука, М., 2006.

[2] В. П. Маслов, Матем. заметки, 64:5 (1998), 791-792.

[3] В. П. Маслов, Матем. заметки, 81:2 (2007), 251-264.

[4] В. П. Маслов, Докл. РАН, 411:5 (2006), 591-593.

[5] V.P. Maslov, Russ. J. Math. Phys., 12:4 (2005), 483-488.

[6] В. П. Маслов, Матем. заметки, 80:2 (2006), 220-230.

[7] V.P. Maslov, Russ. J. Math. Phys., 14:1 (2007), 31-60. 